\title{
Integrando Álgebra, Arte e Geometria com o Software Graphequation
}

\author{
Eleni Bisognin-UNIFRA - eleni @unifra.br \\ Maria do Carmo Barbosa Trevisan -UNIFRA- mcbtrevisan@ hotmail.com \\ Vanilde Bisognin-UNIFRA-vanilde@unifra.br
}

\section{RESUMO}

O presente trabalho descreve resultados parciais de uma pesquisa que tem como foco as Tecnologias da Informação e Comunicação e sua utilização no ensino e aprendizagem da Matemática. Os resultados fazem parte do trabalho desenvolvido com professores da Educação Básica e alunos do curso de Licenciatura em Matemática, que tem por objetivo, integrar alunos e professores em um trabalho pedagógico colaborativo com o uso das TICs. Por meio das atividades desenvolvidas com o software GraphEquation, são explorados os conceitos e propriedades de funções, equações e inequações, que permitiram integrar conteúdos de Álgebra, Geometria e Arte. Os resultados evidenciaram que, por meio da visualização gráfica que o software oferece, é possível construir novos conceitos matemáticos relacionados com o conceito de função.

Palavras-chave: recursos computacionais; funções; equações e inequações; ensino e aprendizagem de matemática.

\section{Integrating Algebra, Art and Geometry whit the software Graphequation}

\begin{abstract}
This paper describes partial results of a research that focuses on the Technologies of Information and Communication and its use in teaching and learning of mathematics. The results are part of the work made with teachers of Basic Education and students of Mathematics, which aims to integrate students and teachers in a collaborative work with the pedagogical use of ICTs. Through the activities developed with the software GraphEquation, concepts and properties of functions, equations and inequalities are explored, which enabled the integration of content of Algebra, Geometry and Art. The results showed that, by viewing graphical that the software offers, it is possible to construct new mathematical concepts related to the concept of function
\end{abstract}

Key-words: computer resources; functions; equations and inequalities; teaching and learning of mathematics. 


\section{1-INTRODUÇÃO}

Nos últimos anos, tem-se evidenciado o crescimento de propostas pedagógicas que integram as tecnologias da informação e comunicação no ensino de Matemática. Estas tecnologias envolvem uso de vídeos, blogs, chats, hipertexto, uso de softwares computacionais, entre outros. Resultados de pesquisas encontrados em Valente (1999) têm mostrado que estes recursos, quando bem utilizados, podem contribuir para tornar o ensino, principalmente o de Matemática, mais atraente e motivador, e dessa forma, trazer uma colaboração significativa para a aprendizagem.

De acordo com o que consta no National Council of Teachers of Mathematics (2000, apud, NETO e MAIA, 2009, p.1),

[...] a tecnologia é essencial no ensino e na aprendizagem da Matemática; influencia a Matemática que é ensinada e melhora a aprendizagem dos alunos. Os estudantes podem aprender mais Matemática e com mais profundidade, utilizando a tecnologia de forma adequada e responsável (...). Alguns estudantes podem se beneficiar de situações mais simples e cativantes proporcionadas pelos computadores. (2009,p.1).

A introdução do computador na educação não deve consistir apenas numa atualização tecnológica, mas é importante que seja utilizado efetivamente no processo de desenvolvimento da capacidade de criar e pensar, além de ser usado como uma ferramenta que facilita a criação de novos conhecimentos e, de acordo com Valente (1999), que promova a aprendizagem ao invés do ensino, que o aprendiz participe controle seu processo de aprendizagem e, ainda, que auxilie o professor a entender que a educação é um processo de construção de conhecimento pelo aluno e não somente a transferência de conhecimento.

O trabalho aqui relatado faz parte de um projeto de pesquisa que envolve professores da Educação Básica e alunos do curso de Licenciatura em Matemática com a finalidade de discutir, propor e avaliar diferentes estratégias de ensino com o uso de tecnologias da informação e comunicação.

As atividades criadas no âmbito do programa "Oficinas Pedagógicas" que integra professores da Educação Básica e licenciandos, teve como foco a integração da Álgebra, Geometria e Arte e, neste percurso, optou-se pelo uso do software GraphEquation. Considera-se que esta estratégia integrando professores com experiência e alunos em formação inicial, utilizando recursos computacionais, pode tornar o estudo da Matemática mais atraente e as aulas mais ativas e participativas.

\section{2- O CONTEXTO DO TRABALHO}

Desde 2004 tem-se desenvolvido um programa que integra professores da Educação Básica, professores do curso de Licenciatura em Matemática e alunos desse curso com o objetivo de qualificar a formação inicial dos alunos e de possibilitar a formação continuada dos docentes em serviço. Nos últimos anos os estudos tiveram como foco as Tecnologias da Informação e Comunicação e seu uso na sala de aula. Esse estudo tem se realizado em grupos colaborativos que se reúnem quinzenalmente. Nesses 
encontros são abordadas questões metodológicas; proposição de atividades com o uso das TICs para diferentes níveis de ensino; análise das potencialidades e fragilidades na aplicação das atividades e avaliação; organização de cursos de qualificação pedagógica ofertados para professores em forma de oficinas.

Neste processo de integração entre alunos em formação inicial e professores em formação continuada, a preocupação central é com a elaboração de propostas pedagógicas e sua incorporação no dia a dia do trabalho em sala de aula, envolvendo as TICs e discutir o papel do professor em relação aos avanços tecnológicos que desafiam o fazer pedagógico.

Entendemos que, em relação às TICs, o professor precisa ter competência para elaboração de materiais didáticos, conhecer ferramentas que viabilizem estes materiais e vivenciem todo processo de seu uso, como aluno. É necessário que os professores possuam saberes, mas também competências profissionais, que vão além dos conteúdos que precisam ensinar para que o trabalho de sala de aula, envolvendo as TICs, traga uma contribuição efetiva para o ensino e aprendizagem da Matemática.

De acordo com Ponte et.al (1998),

\begin{abstract}
A tecnologia tem um enorme potencial a serviço da renovação dos processos de ensino e aprendizagem. O desenvolvimento da autonomia e responsabilidade dos alunos, bem como seu envolvimento em tarefas de resolução de problemas e noutras atividades criativas, pode ser potencializada pelo uso da tecnologia. Além disso, a tecnologia constitui um suporte valioso na apropriação, por parte dos alunos, de ideias poderosas e processos fundamentais em diversas áreas da Matemática, designadamente no âmbito da Geometria e das funções. (p.107).
\end{abstract}

O conceito de função, que é introduzido no ensino fundamental, é essencial para a construção de outros conceitos, ou seja, muitos outros conceitos avançados são construídos sob sua ótica. No entanto, apesar de ser um conteúdo trabalhado desde a $8^{\text {a }}$ série do ensino fundamental, muitos estudantes apresentam dificuldades na aprendizgem desse conteúdo como atestam as pesquisas de Vinner (1983), Ponte (1992), Clement (2001), Cury (2004), Costa e Igliori (2006), Bisognin et al.(1010), entre outros. Entende-se que as dificuldades encontradas pelos estudantes estão relacionadas, em grande parte, com o modo como este conteúdo é trabalhado pelos professores na sala de aula que, muitas vezes, apresentam de modo abstrato, sem levar em consideração suas múltiplas representações.

A experiência aqui relatada mostra atividades criadas pelos participantes do programa com o software GraphEquation. Este programa permite a construção de funções expressas em forma de equações e inequações e criar diferentes figuras de acordo com a imaginação e criatividade de cada aluno. Enquanto os alunos e professores desenharam figuras eles construíram novos conceitos ou consolidaram os já estudados e, ao mesmo tempo, descobriram diferentes propriedades das funções.

Os resultados relatados tiveram como base a análise das produções escritas pelos quinze participantes das oficinas pedagógicas, realizadas no ano de 2009 sobre o conteúdo de funções e utilizando o software GraphEquation para suas representações. Justifica-se a escolha do tema em face dos resultados das pesquisas citadas anteriormente e pelo fato de que consideramos importante que alunos e professores de Matemática em exercício conheçam os conteúdos que irão trabalhar, pois as 
dificuldades apresentadas pelos docentes em relação a um determinado tópico, podem acarretar problemas na compreensão dos conceitos por parte de seus alunos.

\section{3 - DESCRIÇÃO DA EXPERIÊNCIA}

Para o trabalho de sala de aula foi proposto uma sequência de atividades com o uso do software GraphEquation, com o objetivo de explorar os conceitos e propriedades de funções.

O GraphEquation é um software utilizado para plotagem de gráficos de funções representadas em forma de equações em coordenadas cartesianas ou polares e de inequações. Ele permite trabalhar diferentes conceitos algébricos e geométricos de forma interativa sendo a parte gráfica uma grande auxiliar para compreensão dos conceitos matemáticos. As igualdades e desigualdades algébricas devem ser escritas de acordo com o formalismo do programa sendo possível atribuir valores às variáveis definidas e podendo gerar até quinze gráficos simultâneos, visualizando-os sobrepostos em diferentes cores. Alterando os dados, gerando novos gráficos, analisando as representações e transformações, os alunos podem estabelecer novas relações e assim gerar novos conhecimentos.

A escolha do software foi pautada nas recomendações de Gravina e Santarosa (1998), de que a escolha de um software educacional para o ensino e aprendizagem da Matemática deve levar em conta a criação de um ambiente que coloca o aluno no papel de ativo aprendiz.

A experiência foi desenvolvida em três sessões com duração de duas horas com objetivos bem definidos.

A primeira sessão iniciou-se com a demonstração dos procedimentos básicos para o conhecimento dos principais comandos e funções do software, necessários para o desenvolvimento das atividades.

No decorrer da realização da sessão, todas as tarefas propostas tiveram uma componente prática, que foi materializada em construções geométricas e outra de exploração e investigação dos conteúdos envolvidos. As etapas de cada atividade foram realizadas passo a passo pela professora responsável pela oficina através de vídeo projeção e acompanhados pelos alunos. Assim, os alunos, primeiramente observaram um exemplo para em seguida executarem a atividade proposta e por último, realizarem uma tarefa de forma autônoma que permitiu a consolidação da aprendizagem.

Iniciou-se com a exploração da função linear e da função afim procurando estabelecer a diferença entre elas e analisando as propriedades a partir da variação dos parâmetros. Esta análise foi realizada através da representação algébrica e geométrica e representadas nas Figuras 1 e 2, a seguir.
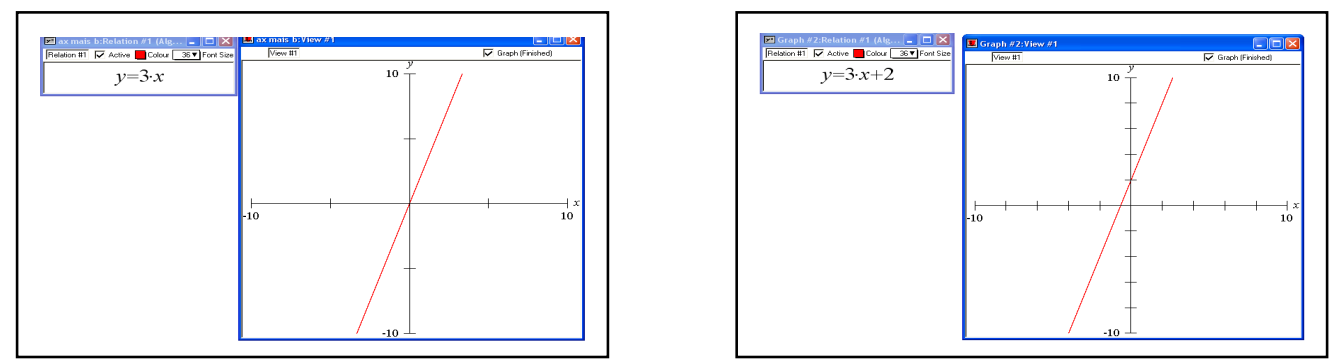
Ao término da atividade verificou-se que os alunos compreenderam os conceitos relacionados com estes tipos de funções, pois conseguiram, de forma autônoma, realizar outras representações fazendo variar os parâmetros $a$ e $b$ da equação $y=a x+b$.

A partir dessas atividades realizou-se um momento de reflexão e investigação em que os alunos descreveram cada uma de suas representações, compartilhando com os colegas da turma o trabalho realizado.

Como segunda atividade, construiu-se o gráfico da função modular explorando o conceito de translação, conforme mostrado nas Figuras 3 e 4.

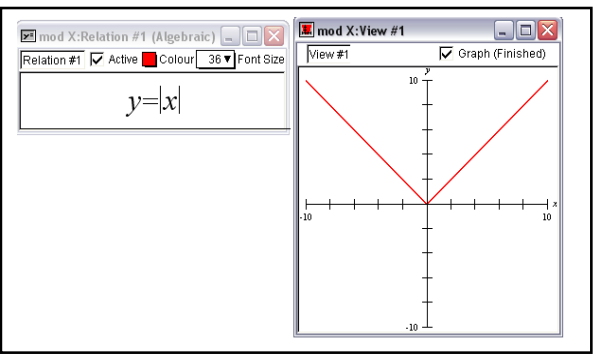

Figura 3 - Gráfico da função modular

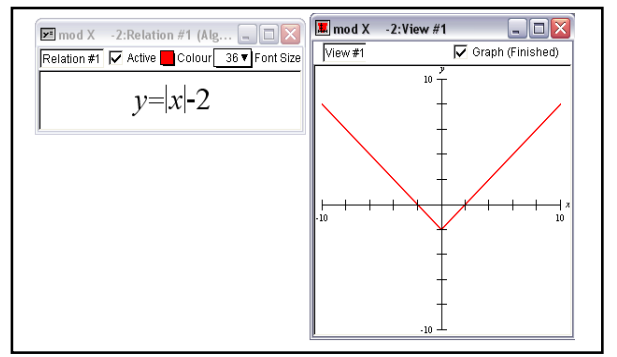

Figura 4 - Gráfico da função modular transladada

$\mathrm{Na}$ terceira parte desta sessão foram propostas atividades cujas representações propiciaram explorar os conceitos de rotação, reflexão, dilatação e contração. As representações das figuras propostas estão descritas nas Figuras 5, 6, 7 e 8.

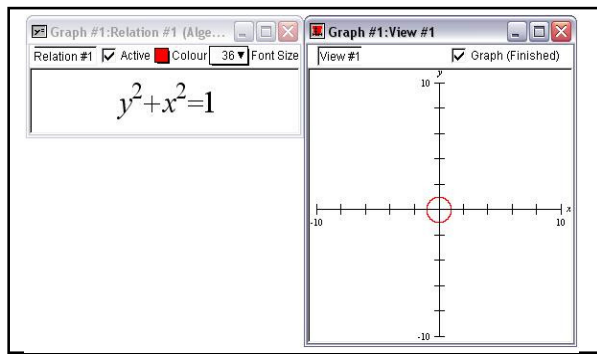

Figura 5 - Círculo centrado na origem

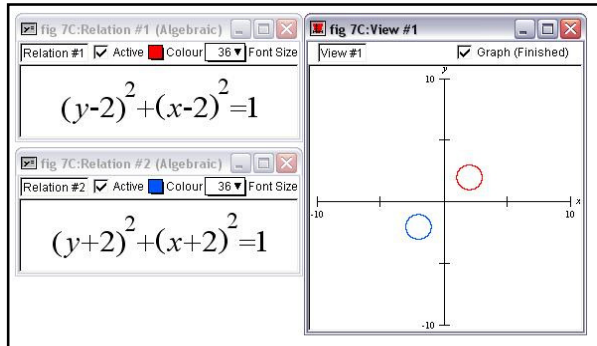

Figura 7 - Reflexão em relação à origem

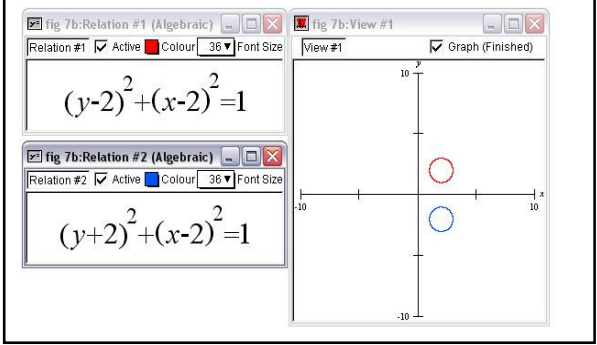

Figura 6 - Reflexão em relação ao eixo x

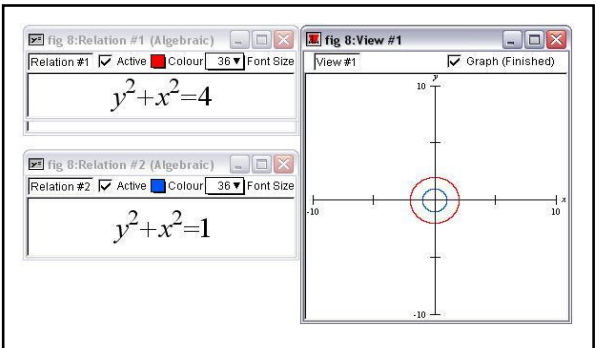

Figura 8 - Dilatação

A partir dos gráficos traçados e das representações algébricas associadas explorou-se as transformações e os conceitos relacionados em cada uma delas. Encerrou-se a sessão propondo aos alunos que realizassem uma tarefa livre que incluísse os conceitos trabalhados. Nessa etapa os alunos propuseram outras figuras, 
como círculo, parábola, hipérbole, quadrado, retângulo, triângulo e exploraram os conceitos estudados anteriormente. As Figuras 9 e 10, a seguir, foram apresentadas pelos grupos A e B.

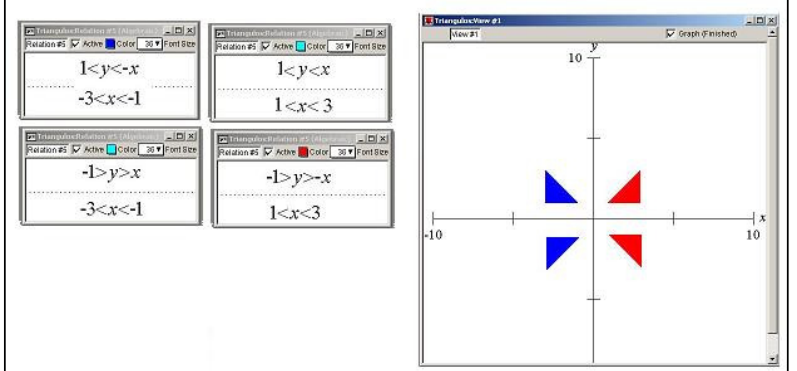

Figura 9 - Reflexão em relação aos eixos

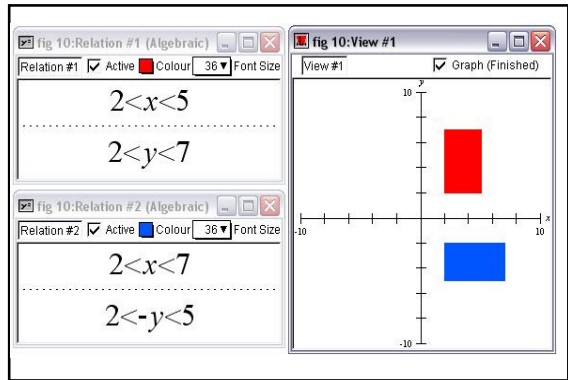

Figura10 - Rotação e translação

A partir das propostas dos alunos foram explorados os polígonos regulares, irregulares e suas diferenças e as relações entre os elementos dos polígonos construídos.

$\mathrm{Na}$ segunda sessão foram propostas atividades envolvendo funções polinomiais de $2^{\circ}$ e $3^{\circ}$ graus, funções trigonométricas, funções logarítmicas e exponenciais e a composição de funções como mostradas nas Figuras 11,12,13 e 14 a seguir.

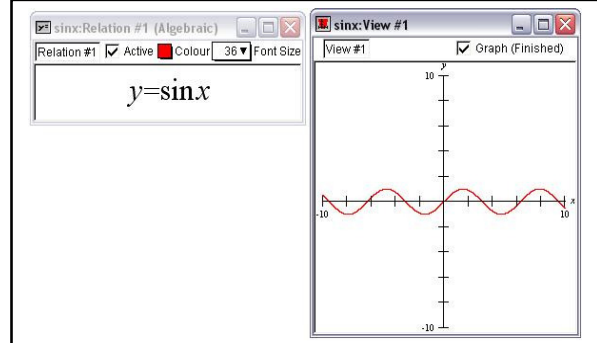

Figura 11 - Gráfico da função seno

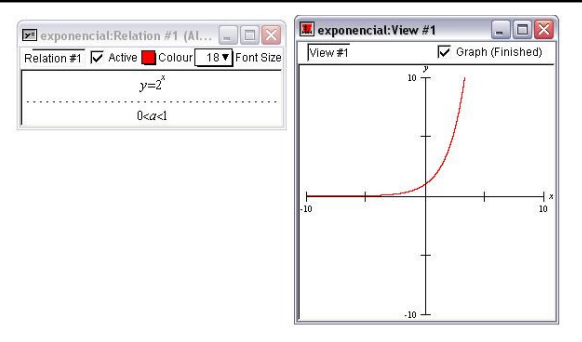

Figura 13 - Gráfico da função exponencial

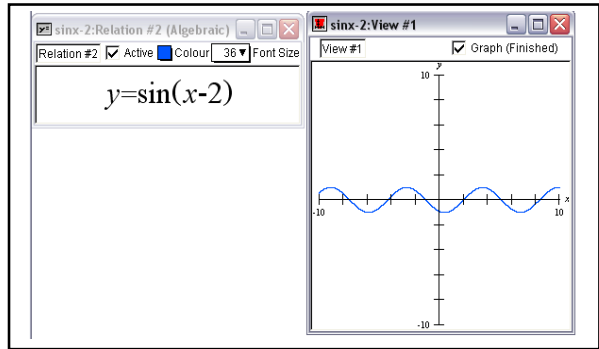

Figura 12 - Translação da função seno

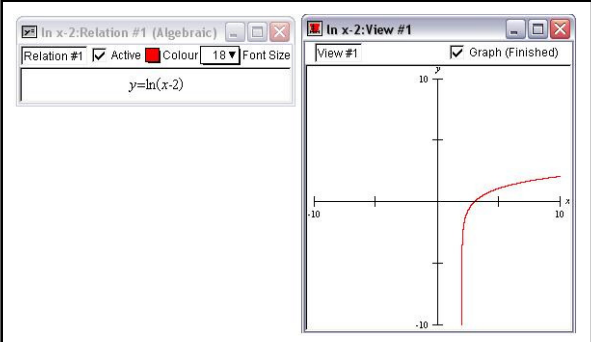

Figura 14 - Gráfico da função logarítmica translada

Nesta sessão observou-se que a maioria dos alunos apresentou dificuldades quanto aos conceitos relacionados com a translação, rotação e reflexão. 
A abordagem das funções por meio da representação geométrica, com auxílio do GraphEquation permitiu a compreensão dos conceitos e propriedades também do ponto de vista algébrico. Ao final da sessão todos os alunos atingiram os objetivos previstos e conseguiram concluir as propriedades algébricas das funções por meio da representação geométrica.

Na terceira e última sessão os alunos foram convidados a fazerem a construção de uma composição de figuras geométricas usando os conceitos e propriedades das figuras estudadas nas sessões anteriores, que fossem interessantes e motivadoras e que usassem a imaginação e criatividade. Alguns alunos realizaram trabalhos considerados excelentes o que mostrou que os conceitos foram compreendidos e que o software GraphEquation revelou-se uma ferramenta eficaz para essa compreensão.

As Figuras 15, 16, 17 e 18 a seguir, mostram algumas dessas construções.

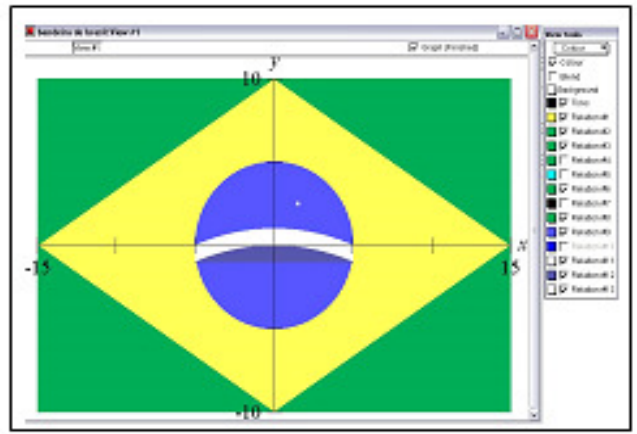

Figura 15 - Construção do aluno A

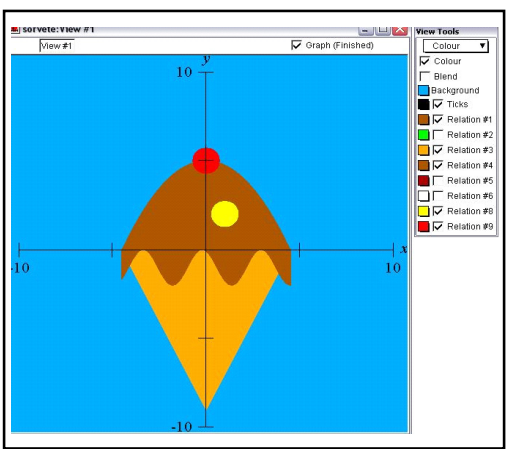

Figura 17: Construção do aluno C

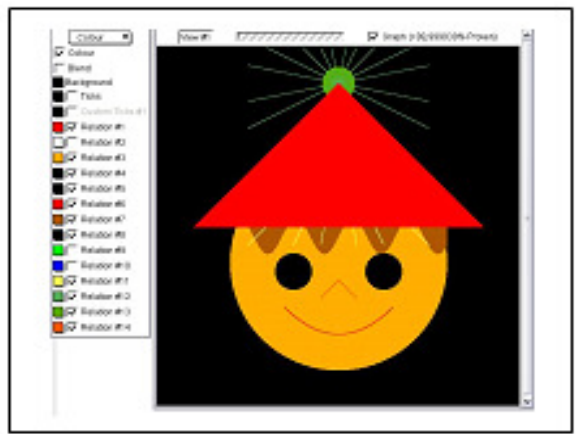

Figura 16 - Construção do aluno B

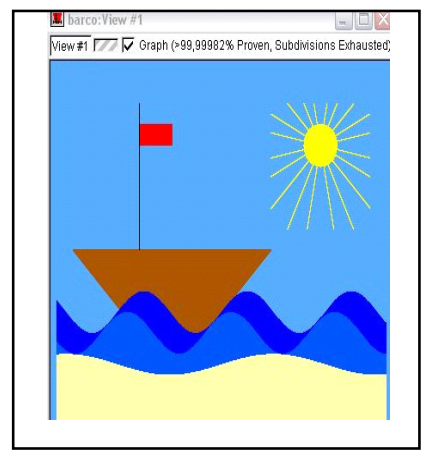

Figura 18: Construção do aluno D

A ação investigativa desencadeada a partir da análise da produção dos alunos propiciou aulas dinâmicas, com muitas explorações, onde os alunos e professores envolveram-se na descoberta e nas representações, utilizando suas próprias formas de produzir. A partir das diferentes representações exploraram-se os conceitos e propriedades de funções e os conceitos de reflexão, translação e rotação.

As atividades descritas são apenas algumas das que foram construídas durante o desenvolvimento das oficinas. As conclusões sobre a eficácia da utilização de recursos computacionais no desenvolvimento das atividades, foram elaboradas a partir da análise dos caminhos percorridos pelos alunos na busca de solução das atividades propostas. 


\section{4 - CONSIDERAÇÔES FINAIS}

Neste trabalho teve-se como propósito descrever os resultados de uma experiência que envolveu alunos em formação inicial e professores em formação continuada, com o uso do GraphEquation, para representação gráfica das funções e seu estudo em sala de aula.

Da análise da produção dos alunos é possível inferir que o software utilizado trouxe uma colaboração efetiva para a construção dos conceitos relacionados com funções e a descoberta de propriedades de figuras planas por meio da integração da representação algébrica e geométrica.

Pôde-se perceber que os alunos conseguiram integrar a Álgebra, a Geometria e a Arte com destaque para as cores e a composição geométrica. Destaca-se também, a conveniência da escolha do software, pois, seu uso permitiu a criação de um ambiente interativo entre os alunos e o conhecimento, o qual só é viável com o auxílio de ferramentas computacionais.

\section{5-REFERÊNCIAS BIBLIOGRÁFICAS}

BISOGNIN,E.; CURY,H.N.; BISOGNIN,V.; Conhecimentos de Professores da Educação Básica sobre o Conceito de Função.In: ENCONTRO NACIONAL DE EDUCAÇÃO MATEMÁTICA,10, 2010, Salvador.Anais...Salvador:SBEM, 2010.1CDROM.

CLEMENT, L. What do students really know about functions? Mathematics Teacher, v. 94, n. 9, p. 745-748, Dec. 2001.

COSTA, A. C.; IGLIORI, S. B. Conhecimentos de estudantes universitários sobre o conceito de função. In: SEMINÁRIO INTERNACIONAL DE PESQUISA EM EDUCAÇÃo MÁTEMÁtICA, 3., 2006, Águas de Lindóia. Anais... Recife: SBEM, 2006. 1 CD-ROM.

CURY, H. N. "Professora, eu só errei um sinal!": como a análise de erros pode esclarecer problemas de aprendizagem. In: CURY, H. N. (Org.). Disciplinas matemáticas em cursos superiores: reflexões, relatos, propostas. Porto Alegre: EDIPUCRS, 2004. p. 111-138.

GRAVINA, Maria Alice; SANTAROSA, Lucila Maria. A aprendizagem Matemática em Ambientes Informatizados. In: CONGRESO IBEROAMERICANO DE INFORMÁTICA EDUCATIVA, 4, 1998, Brasília, Anais...Brasília, 1998, v. 1, p. 2535.

NETO,M.; MAIA,J.S.; Uma experiência de Abordagem Dinâmica da Geometria com a Álgebra no $4^{\circ}$ Ano de Escolaridade.IN: ENCONTRO NACIONAL DE PROFESSORES DE MATEMÁTICA, ProfMat, 2009, Elvas. Anais... Elvas, Portugal, 2009,1 CD ROM.

PONTE, J. P. The history of the concept of function and some educational implications. The Mathematics Educator, v. 3, n. 2, p. 3-8, 1992. 
PONTE,J.; MATOS,J.; ABRANTES,P.; Investigação em Educação matemática: implicações curriculares.Lisboa.Instituto de Inovação Educacional, 1998

VALENTE,J.A.; Computador na sociedade do conhecimento. Campinas, SP : Nied , 1999, 156p.

VINNER, S. Concept definition, concept image and the notion of function. Int. J. Math. Educ. Sci. Technol., v. 14, n. 3, p. 293-305, 1983. 